\title{
Improving Episodes: An Editorial Office perspective
}

The Editorial Office is constantly trying to make improvements in the contents of the journal and how these contents are presented to you, our disciminating readership. In 1997, when the Episodes' office was moved from the U.K. to China, all our efforts were concentrated on restoring the publication schedule from three times per year to the original four (March, June, September, and December) and to restoring the size of each issue from 48 to approximately 80 pages. When this was accomplished in 1998, the focus of attention in the Editorial Office then switched to improving the quality of the contributions and the quality of printing, and we are continuing to forge ahead in addressing these matters.

Regarding the quality of contributions, we have been greatly assisted by the appointment of an Editorial Board, consisting of twenty one internationally based earth scientists who have attained widespread recognition in their fields. The Board assists the Editor by assessing contributed articles and reviews and making recommendations on their suitability for the journal. Together too, the Editor and Associate Editors are striving to increase the content of high quality articles and reviews and to ensure that these deal with notable accomplishments in the earth sciences from the world at large. In these connections, observant readers may notice in this issue a change in the subtitle of the journal, advocated by the IUGS Executive Committee, from "International Geoscience Newsmagazine" to "Journal of International Geoscience". This change does not imply any change in editorial policy. Rather, it is designed to make the name of the journal conform better to existing policy. Another change, one of a technical nature, that readers will see in this issue is a conversion to the use of art-quality paper in order to improve the standard of reproduction of illustrations and, we hope, to pave the way for the accommodation of a higher proportion of coloured illustrations in the future.

Since the birth of the journal in 1978, many readers have regarded Episodes as the principal source of information about our organizational publisher, IUGS. Despite the change in the journal's subtitle, this role is being maintained undi- minished, and, indeed, the Editorial Office is striving to expand it, with more progress reports on IUGS's scientific programs and those it operates jointly with other agencies, and with more contributions from its affiliated organizations. Furthermore, the journal will continue to publish Conference Reports on important meetings of international interest, reviews of appropriate new books and maps, and the IUGS five-year calendar of forthcoming events. Episodes maintains close liaison with the IUGS world-wide website: http://www.iugs.org.

The IUGS Advisory Board for Publications has long believed Episodes to be a particularly suitable medium for the publication of reviews of topical subjects in the earth sciences. These contributions tend to be longer and more timeconsuming to prepare than articles, but they are a proven means of presenting broad, up-to-date status reports on subjects of widespread importance to the international geoscientific community. One such review appears in this issue. It deals with Canada's highly successful, collaborative, multidisciplinary project, LITHOPROBE, which has developed major new ideas, not only about the Canadian landmass and its off-shore extensions but about the tectonic evolution of the entire North American continent. We hope you will enjoy it.

The Editor and the Editorial Office in Beijing are always interested in hearing from you, our readers, about Episodes. You should feel free to contribute to "Letters to the Editor", or to write to us privately with either compliments or criticisms (address, telephone and fax numbers, and e-mail code are available inside the front cover of each issue). If you prefer to direct such letters to an outside party, they can be sent to the Chairman of the IUGS Advisory Board for Publications (name and address available from either the IUGS www site or the IUGS Directory).

\section{Zhang Hongren \\ Editor}

